\title{
RADIATION EFFECTS ON EXPONENTIALLY ACCELERATED VERTICAL PLATE WITH UNIFORM MASS DIFFUSION
}

\author{
K.E. Sathappan and R. Muthucumaraswamy \\ Department of Applied Mathematics \\ Sri Venkateswara College of Engineering \\ Sriperumbudur - 602 105, India. \\ Phone : +9144-271500, Fax : +9144- 27162462 \\ Email: sathappan@svce.ac.in
}

\begin{abstract}
Thermal radiation effects on unsteady free convective flow of a viscous incompressible flow past an exponentially accelerated infinite isothermal vertical plate with uniform mass diffusion have been studied. An exact solution to the dimensionless governing equations has been obtained by the Laplace transform method. The effects of velocity, temperature and concentration are studied for different parameters like the thermal radiation parameter, Schmidt number, thermal Grashof number, mass Grashof number and time. It is observed that the velocity increases with an increase in the parameter ' $a$ '.
\end{abstract}

Keywords: Radiation, grey, isothermal, vertical plate, heat and mass transfer.

\section{INTRODUCTION}

Radiative heat and mass transfer play an important role in manufacturing industries for the design of fins, steel rolling, nuclear power plants, gas turbines and various propulsion devices for aircraft, combustion and furnace design. If the temperature of the surrounding fluid is rather high, radiation effects play an important role and this situation exists in space technology. In such cases, it is necessary to take into account the combined effect of thermal radiation and mass diffusion. England and Emery (1969) have studied the thermal radiation effects of an optically thin grey gas bounded by a stationary vertical plate. Free convection effects on flow past an exponentially accelerated vertical plate were studied by Singh and Kumar (1984). The skin friction for accelerated vertical plate has been studied analytically by Hossain and Shayo (1986). Jha et al. (1991) analysed mass transfer effects on an exponentially accelerated infinite vertical plate with constant heat flux and uniform mass diffusion.

Das et al. (1996) have analysed radiation effects on flow past an impulsively started infinite isothermal vertical plate. The radiation effect on mixed convection along an isothermal vertical plate was studied by Hossain and Takhar (1996). Raptis and Perdikis (1999) studied the effects of thermal radiation and free convection flow past a moving vertical plate. Muthucumaraswamy et al. (2008) obtained the heat transfer effects on flow past an exponentially accelerated vertical plate with variable temperature.

The governing equations were solved analytically. However, the simultaneous heat and mass transfer effects on exponentially accelerated infinite isothermal vertical plate with uniform mass diffusion in the presence of thermal radiation have not been 
studied in the literature. Hence, it is proposed to study the effects on flow past an exponentially accelerated infinite isothermal vertical plate in the presence of uniform mass diffusion. The dimensionless governing equations are solved using the Laplacetransform technique.

\section{MATHEMATICAL ANALYSIS}

Thermal radiation effects on unsteady flow of a viscous incompressible fluid past an exponentially accelerated infinite isothermal vertical plate with uniform mass diffusion have been studied. Consider the unsteady flow of a viscous incompressible fluid which is initially at rest and surrounds an infinite vertical plate with temperature $T_{\infty}$ and concentration $C_{\infty}^{\prime}$. Here, the $x$-axis is taken along the plate in the vertically upward direction and the $y$-axis is taken normal to the plate. Initially, it is assumed that the plate and the fluid are of the same temperature and concentration. At time $t^{\prime}>0$, the plate is exponentially accelerated with a velocity $u=u_{0} \exp \left(a^{\prime} t^{\prime}\right)$ in its own plane and the temperature from the plate is raised to $T_{w}$ and the concentration level near the plate is also raised to $C_{w}^{\prime}$. The fluid considered here is a grey medium, absorbing-emitting radiation but non-scattering.

Then by the usual Boussinesq approximation, the unsteady flow is governed by the following equations:

$$
\begin{gathered}
\frac{\partial u^{\prime}}{\partial t^{\prime}}=g \beta\left(T-T_{\infty}\right)+g \beta^{*}\left(C^{\prime}-C_{\infty}^{\prime}\right)+v \frac{\partial^{2} u^{\prime}}{\partial y^{\prime 2}} \\
\rho C_{p} \frac{\partial T}{\partial t^{\prime}}=k \frac{\partial^{2} T}{\partial y^{2}}-\frac{\partial q_{r}}{\partial y} \\
\frac{\partial C^{\prime}}{\partial t^{\prime}}=D \frac{\partial^{2} C^{\prime}}{\partial y^{2}}
\end{gathered}
$$

with the following initial and boundary conditions:

$$
\begin{array}{llll}
t^{\prime} \leq 0: & u=0, & T=T_{\infty}, & C^{\prime}=C_{\infty}^{\prime} \text { for all } y \\
t^{\prime}>0: & u=u_{0} \exp \left(a^{\prime} t^{\prime}\right), & T=T_{w}, & C^{\prime}=C_{w}^{\prime} \text { at } y=0 \\
& u=0, & T \rightarrow T_{\infty}, & C^{\prime} \rightarrow C_{\infty}^{\prime} \text { as } y \rightarrow \infty
\end{array}
$$

The local radiant for the case of an optically thin grey gas is expressed by Eq. (5).

$$
\frac{\partial q_{r}}{\partial y}=-4 a^{*} \sigma\left(T_{\infty}^{4}-T^{4}\right)
$$

It is assumed that the temperature differences within the flow are sufficiently small such that $T^{4}$ may be expressed as a linear function of the temperature. This is accomplished by expanding $T^{4}$ in a Taylor series about $T_{\infty}$ and neglecting higher-order terms, thus

$$
T^{4} \cong 4 T_{\infty}^{3} T-3 T_{\infty}^{4}
$$

By using Eq. (5) and Eq. (6), as Eq. (2) reduces to 


$$
\rho C_{p} \frac{\partial T}{\partial t^{\prime}}=k \frac{\partial^{2} T}{\partial y^{2}}+16 a^{*} \sigma T_{\infty}^{3}\left(T_{\infty}-T\right)
$$

On introducing the following dimensionless quantities:

$$
\begin{array}{r}
U=\frac{u}{u_{0}}, t=\frac{t^{\prime} u_{0}^{2}}{v}, Y=\frac{y u_{0}}{v}, \theta=\frac{T-T_{\infty}}{T_{w}-T_{\infty}}, \\
G r=\frac{g \beta v\left(T_{w}-T_{\infty}\right)}{u_{0}^{3}}, C=\frac{C^{\prime}-C_{\infty}^{\prime}}{C_{w}^{\prime}-C_{\infty}^{\prime}}, G c=\frac{v g \beta^{*}\left(C_{w}^{\prime}-C_{\infty}^{\prime}\right)}{u_{0}^{3}}, \\
\operatorname{Pr}=\frac{\mu C_{p}}{k}, S c=\frac{v}{D}, R=\frac{16 a^{*} v^{2} \sigma T_{\infty}^{3}}{k u_{0}^{2}}, a=\frac{a^{\prime} v}{u_{0}^{2}}
\end{array}
$$

in Eq. (1) to Eq. (4), leads to

$$
\begin{gathered}
\frac{\partial U}{\partial t}=G r \theta+G c C+\frac{\partial^{2} U}{\partial Y^{2}} \\
\frac{\partial \theta}{\partial t}=\frac{1}{P r} \frac{\partial^{2} \theta}{\partial Y^{2}}-\frac{R}{P r} \theta \\
\frac{\partial C}{\partial t}=\frac{1}{S c} \frac{\partial^{2} C}{\partial Y^{2}}
\end{gathered}
$$

The initial and boundary conditions in non-dimensional form are

$$
\begin{aligned}
& U=0, \quad \theta=0, \quad C=0, \quad \text { for all } \quad Y, t \leq 0 \\
& t>0: \quad U=\exp (a t), \quad \theta=1, \quad C=1, \quad \text { at } \quad Y=0 \\
& U=0, \quad \theta \rightarrow 0, \quad C \rightarrow 0 \quad \text { as } \quad Y \rightarrow \infty
\end{aligned}
$$

Equations (9) to (11), subject to the boundary conditions (12), are solved by the usual Laplace-transform technique and the solutions are derived as follows:

$$
\begin{gathered}
\theta=\frac{1}{2}\left[\begin{array}{c}
\exp (2 \eta \sqrt{R t}) \operatorname{erfc}(\eta \sqrt{\operatorname{Pr}}+\sqrt{a t}) \\
+\exp (-2 \eta \sqrt{R t}) \operatorname{erfc}(\eta \sqrt{\operatorname{Pr}}-\sqrt{a t})
\end{array}\right] \\
C=\operatorname{erfc}(\eta \sqrt{S c})
\end{gathered}
$$




$$
\begin{aligned}
& U= \frac{\exp (a t)}{2}[\exp (2 \eta \sqrt{a t}) \operatorname{erfc}(\eta+\sqrt{a t})+\exp (-2 \eta \sqrt{a t}) \operatorname{erfc}(\eta-\sqrt{a t})] \\
&-d \exp (c t)[\exp (2 \eta \sqrt{c t}) \operatorname{erfc}(\eta \sqrt{\operatorname{Pr}}+\sqrt{c t})+\exp (-2 \eta \sqrt{c t}) \operatorname{erfc}(\eta \sqrt{\operatorname{Pr}}-\sqrt{c t})] \\
&-e\left[\left(1+2 \eta^{2}\right) \operatorname{erfc}(\eta)-\frac{2 \eta}{\sqrt{\pi}} \exp \left(-\eta^{2}\right)\right]+2 d \operatorname{erfc}(\eta) \\
&-d[\exp (2 \eta \sqrt{R t}) \operatorname{erfc}(\eta \sqrt{\operatorname{Pr}}+\sqrt{b t})+\exp (-2 \eta \sqrt{R t}) \operatorname{erfc}(\eta \sqrt{\operatorname{Pr}}-\sqrt{b t})] \\
&+d \exp (c t)[\exp (-2 \eta \sqrt{\operatorname{Pr}(b+c) t}) \operatorname{erfc}(\eta \sqrt{\operatorname{Pr}}-\sqrt{(b+c) t}) \\
&+\exp (2 \eta \sqrt{\operatorname{Pr}(b+c) t}) \operatorname{erfc}(\eta \sqrt{\operatorname{Pr}}+\sqrt{(b+c) t})] \\
&+ e\left[\left(1+2 \eta^{2} S c\right) \operatorname{erfc}(\eta \sqrt{S c})-\frac{2 \eta \sqrt{S c}}{\sqrt{\pi}} \exp \left(-\eta^{2} S c\right)\right]
\end{aligned}
$$

where $b=\frac{R}{P r}, c=\frac{R}{1-P r}, d=\frac{G r}{2 c(1-P r)}, e=\frac{G c t}{(1-S c)}$ and $\eta=\frac{Y}{2 \sqrt{t}}$.

\section{RESULTS AND DISCUSSION}

For physical understanding of the problem numerical computations have been carried out for different physical parameters $R, a, G r, G c, S c$ and $t$ upon the nature of the flow and transport. The value of the Schmidt number $S c$ is taken to be 0.6 , which corresponds to water-vapour. Also, the value of Prandtl number $\mathrm{Pr}$ is chosen such that it represents air $(\operatorname{Pr}=0.71)$. The numerical values of the velocity are computed for different physical parameters like $a$, the thermal radiation parameter, Prandtl number, thermal Grashof number, mass Grashof number, Schmidt number and time.

The velocity profiles for different values of $(a=0,0.5,1), G r=G c=5$ and $R=2$ at time $t=0.2$ are presented in Figure 1. It is observed that the velocity increases with increasing values of $a$. The effect of velocity profiles for different time ( $t=0.4,0.6,0.8), R=2, a=0.5, G r=5$ and $G c=5$ are shown in Figure 2. In this case, the velocity increases gradually with respect to time $t$. The effect of velocity for different values of the Schmidt number $(S c=0.16,0.3,0.6,0.78)$ and time $t=0.2$ are shown in Figure 3. The trend shows that the velocity increases with decreasing Schmidt number. The relative variation of the velocity with the magnitude of the time and the Schmidt number is observed. The velocity profiles for different thermal Grashof numbers $(G r=2,5)$, mass Grashof numbers $(G c=5,10), a=0.5$ and $R=2$ and time $t=0.2$ are shown in Figure 4. It is clear that the velocity increases with an increasing thermal Grashof number or mass Grashof number. 


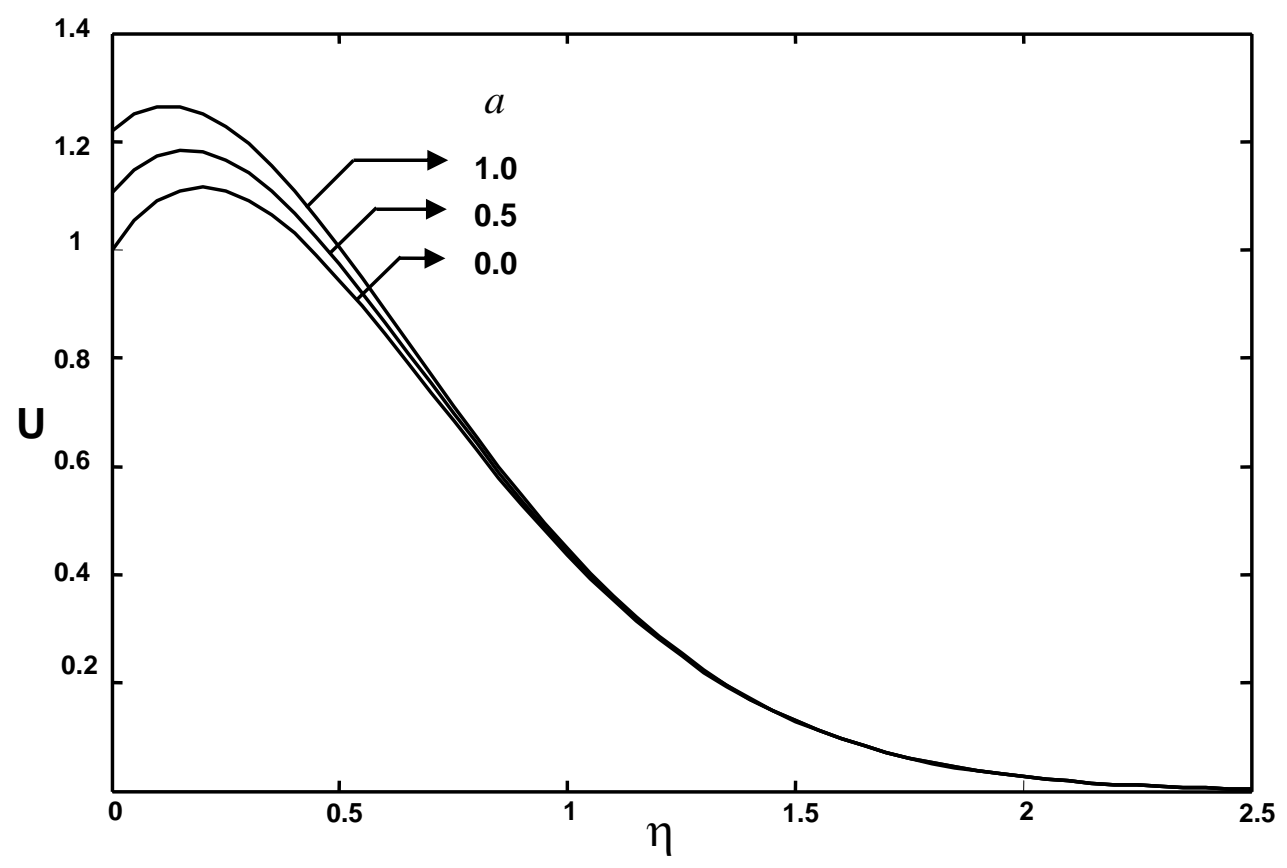

Figure 1. Velocity profiles for different $a$

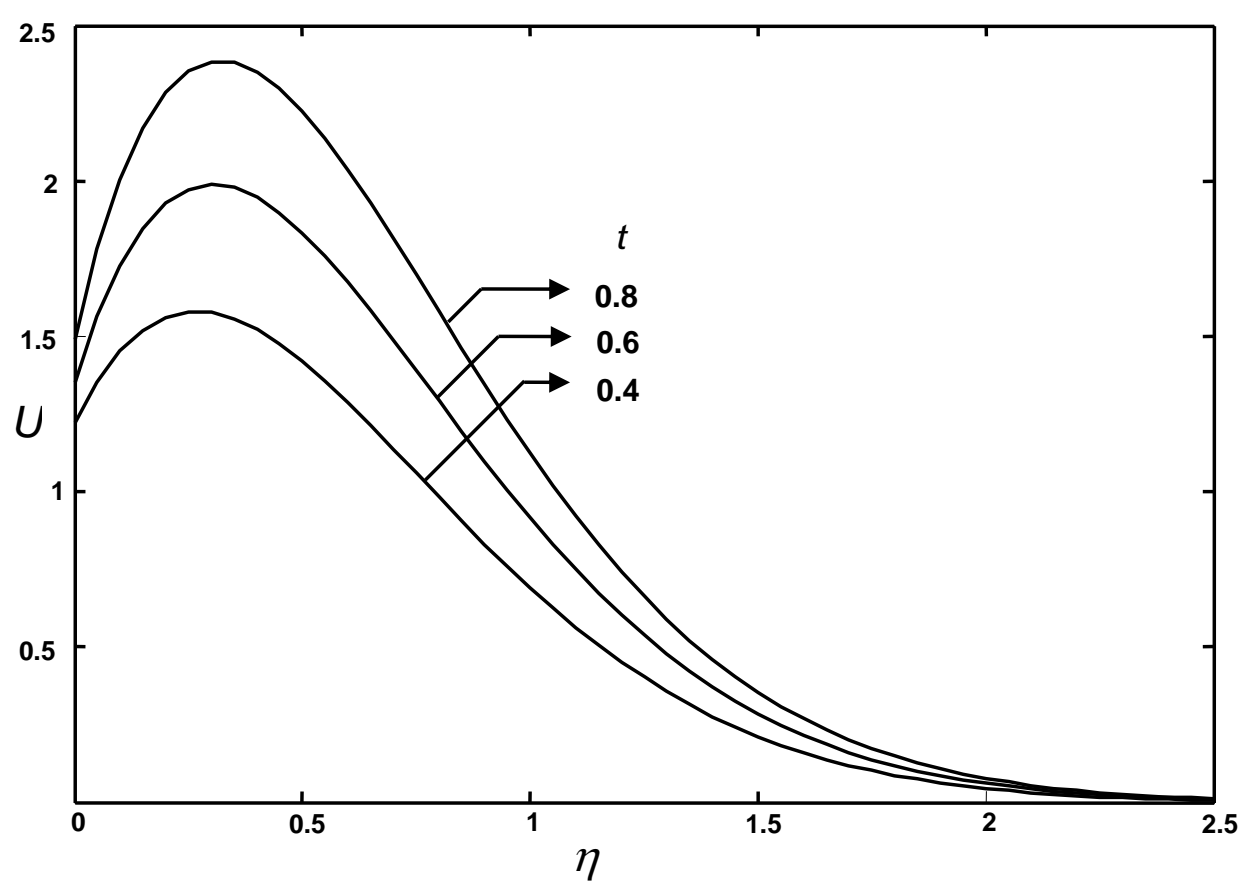

Figure 2. Velocity profiles for different $t$ 


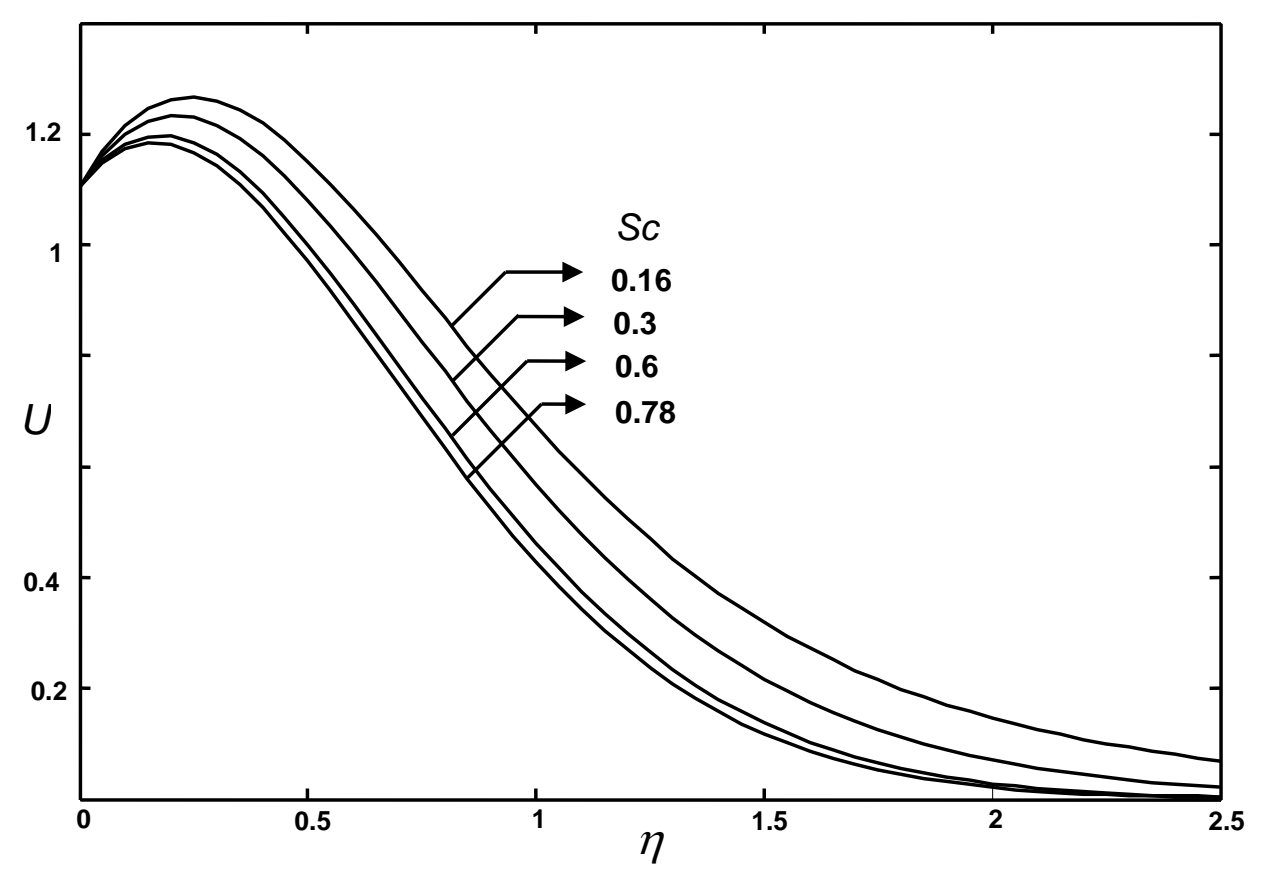

Figure 3. Velocity profiles for different Sc

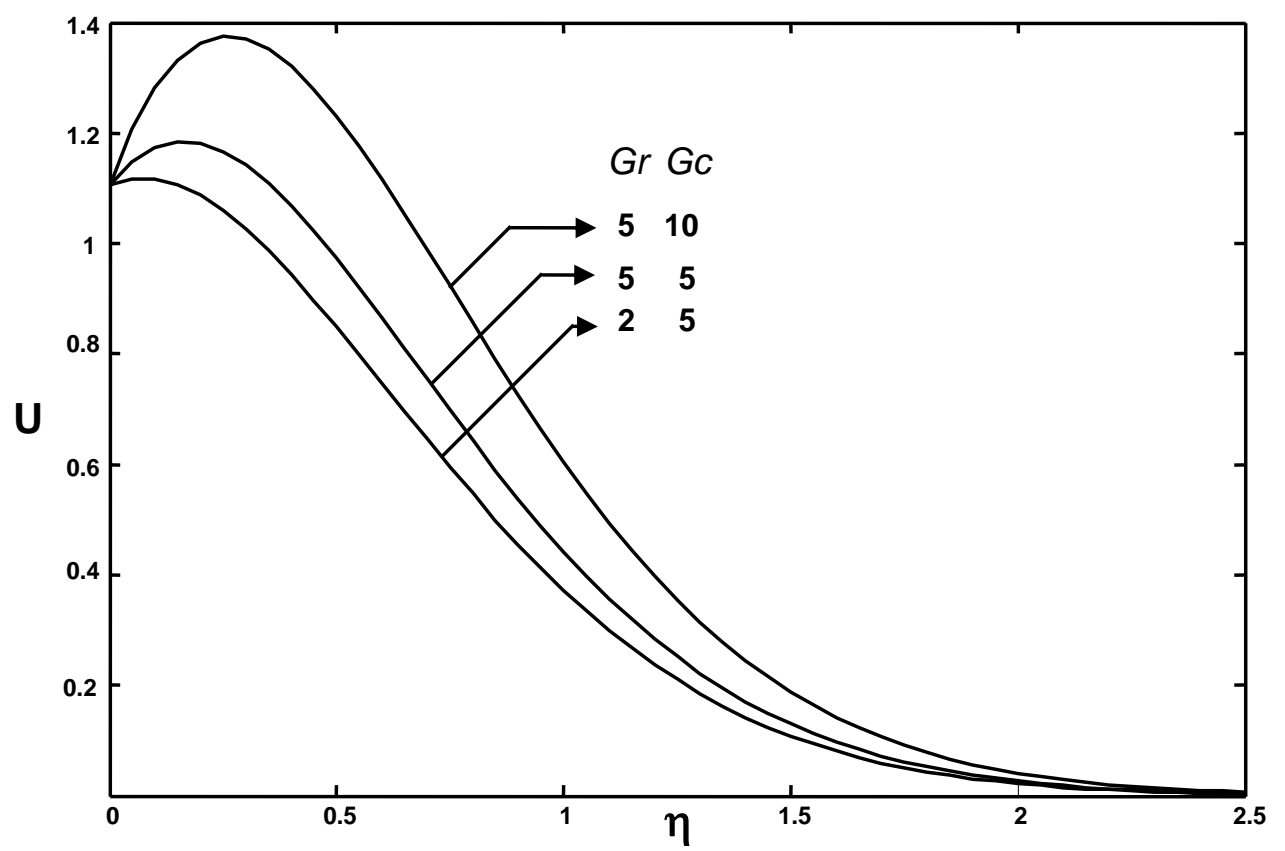

Figure 4. Velocity profiles for different $\mathrm{Gr}$ and Gc

The effect of velocity for different values of the radiation parameter ( $R=2,5,10$ ), $a=0.5, G r=G c=5, R=2$ and $t=0.4$ is shown in Figure 5. The trend shows that the velocity increases with decreasing radiation parameter. It is observed that the velocity decreases in the presence of high thermal radiation. The temperature profiles calculated for different values of the thermal radiation parameter $(R=0.2,2,5)$ and time $(t=0.2,0.6)$ are shown in Figure 6 for air $(P r=0.71)$. The effect of the thermal radiation parameter is important in temperature profiles. It is observed that the 
temperature increases with decreasing radiation parameter. The trend is just reversed with respect to time $t$. Figure 7 represents the effect of concentration profiles for different Schmidt numbers $(S c=0.3,0.6,0.78,2.01)$. The effect of concentration is important in the concentration field. The profiles have the common feature that the concentration decreases in a monotone fashion from the surface to a zero value far away in the free stream. It is observed that the wall concentration increases with decreasing values of the Schmidt number.

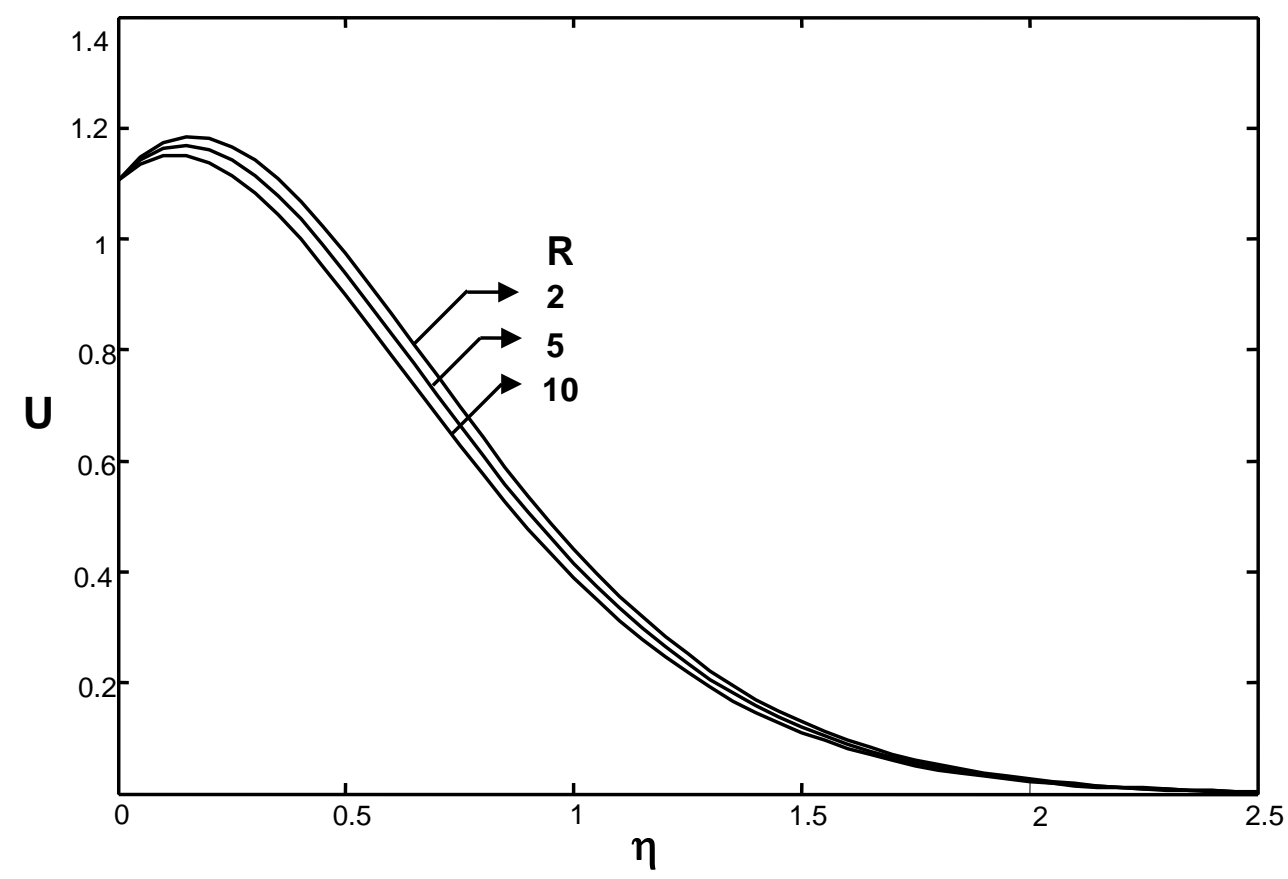

Figure 5. Velocity profiles for different $\mathrm{R}$

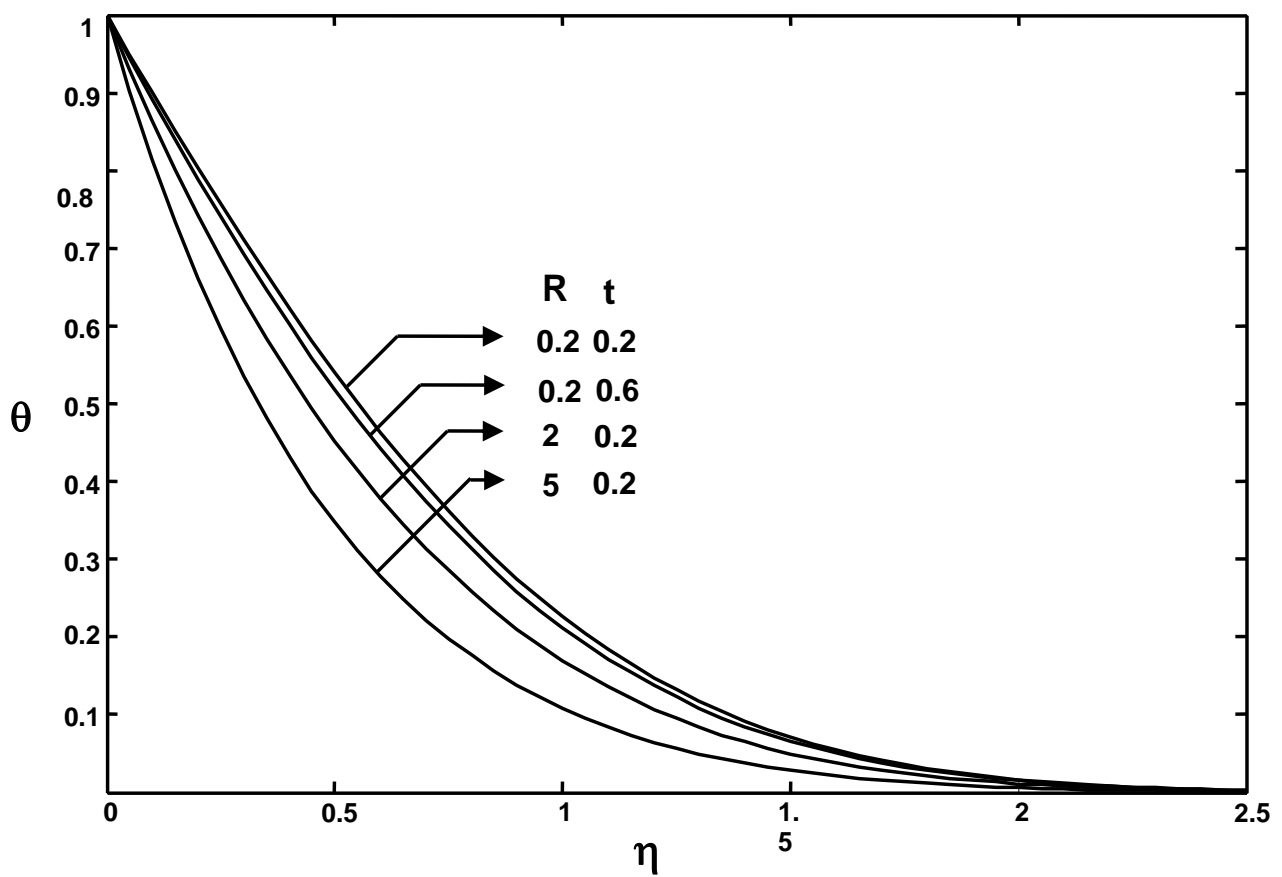

Figure 6. Temperature profiles for different $\mathrm{R}$ and $\mathrm{t}$ 


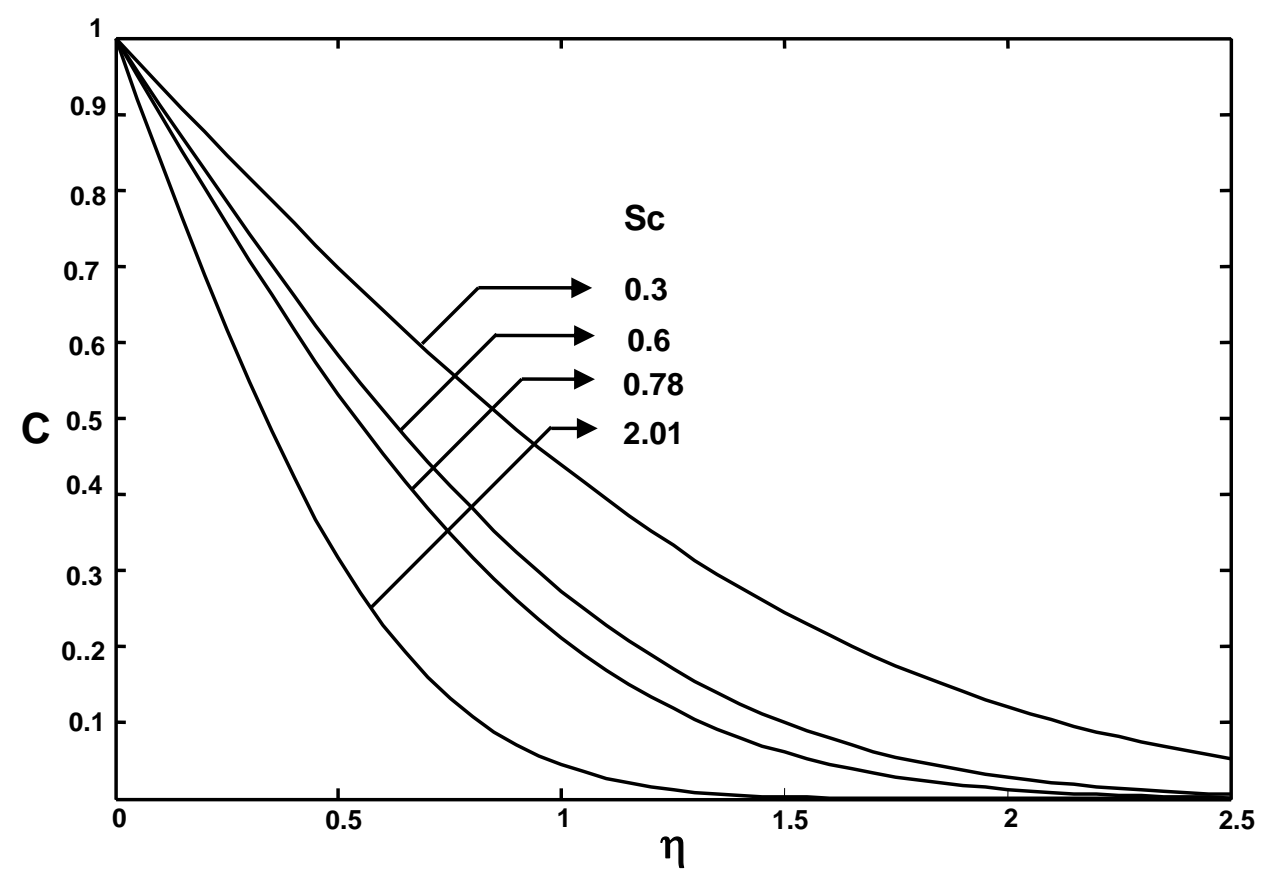

Figure 7. Concentration profiles for different Sc

\section{CONCLUSIONS}

Thermal radiation effects on unsteady flow past an exponentially accelerated infinite isothermal vertical plate, in the presence of uniform wall concentration, are studied. The dimensionless equations are solved using the Laplace transform technique. The effect of velocity, temperature and concentration for the different parameters $a, R, G r, G c, S c$ and $t$ are studied. The conclusions of the study are as follows:

1. The velocity increases with an increase in parameter $a$ and $G r$ or $G c$ and the trend is reversed with respect to the radiation parameter $R$ and Schmidt number Sc.

2. The temperature decreases due to high thermal radiation.

3. It is observed that the concentration increases with decreasing Schmidt number.

\section{REFERENCES}

Jha, B.K., Prasad, R. and Rai, S. 1981. Mass transfer effects on the flow past an exponentially accelerated vertical plate with constant heat flux. Astrophysics and Space Science, 181, 125-134.

Das, U.N., Deka, R.K. and Soundalgekar, V.M. 1996. Radiation effects on flow past an impulsively started vertical infinite plate. Journal of Theoretical Mechanics, 1: 111-115.

England, W.G. and Emery, A.F. 1969. Thermal radiation effects on the laminar free convection boundary layer of an absorbing gas. Journal of Heat Transfer, 91, 37-44.

Hossain, M.A. and Shayo, L.K. 1986. The skin friction in the unsteady free convection flow past an accelerated plate. Astrophysics and Space Science, $125,315-324$. 
Hossain, M.A. \& Takhar, H.S. (1996) Radiation effect on mixed convection along a vertical plate with uniform surface temperature. Heat and Mass Transfer, 31: 243-248.

Muthucumaraswamy, R., Sathappan, K.E. and Natarajan, R. 2008. Heat transfer effects on flow past an exponentially accelerated vertical plate with variable temperature. Theoretical and Applied Mechanics, 35: 323-331.

Raptis, A. and Perdikis, C. 1999. Radiation and free convection flow past a moving plate. International Journal of Applied Mechanics and Engineering, 4: 817-821.

Singh, A.K. and Kumar, N. 1984. Free convection flow past an exponentially accelerated vertical plate. Astrophysics and Space Science, 98: 245-258.

\section{Nomenclature}

\begin{tabular}{|c|c|}
\hline$A, a, a^{\prime}$ & constants \\
\hline$a^{*}$ & absorption constants \\
\hline$C^{\prime}$ & species concentration in the fluid $\mathrm{kg} \mathrm{m}^{-3}$ \\
\hline$C$ & dimensionless concentration \\
\hline$C_{p}$ & specific heat at constant pressure $J . \mathrm{kg}^{-1} . k$ \\
\hline$D$ & mass diffusion coefficient $m^{2} . s^{-1}$ \\
\hline$G_{c}$ & mass Grashof number \\
\hline$G r$ & thermal Grashof number \\
\hline$g$ & acceleration due to gravity $m \cdot s^{-2}$ \\
\hline$k$ & thermal conductivity $W \cdot m^{-1} \cdot K^{-1}$ \\
\hline$R$ & thermal radiation parameter \\
\hline $\operatorname{Pr}$ & Prandtl number \\
\hline Sc & Schmidt number \\
\hline$T$ & temperature of the fluid near the plate $K$ \\
\hline$t^{\prime}$ & time $s$ \\
\hline$u$ & velocity of the fluid in the $x^{\prime}$-direction $m \cdot s^{-1}$ \\
\hline$u_{0}$ & velocity of the plate $m . s^{-1}$ \\
\hline$u$ & dimensionless velocity \\
\hline$y$ & coordinate axis normal to the plate $m$ \\
\hline$Y$ & dimensionless coordinate axis normal to the plate \\
\hline \multicolumn{2}{|c|}{ Greek symbols } \\
\hline$\beta$ & volumetric coefficient of thermal expansion $K^{-1}$ \\
\hline$\beta^{*}$ & volumetric coefficient of expansion with concentration $K^{-1}$ \\
\hline$\mu$ & coefficient of viscosity Ra.s \\
\hline$v$ & kinematic viscosity $m^{2} \cdot s^{-1}$ \\
\hline$\rho$ & density of the fluid $\mathrm{kg} \cdot \mathrm{m}^{-3}$ \\
\hline$\tau$ & dimensionless skin-friction $\mathrm{kg} \cdot \mathrm{m}^{-1} \cdot \mathrm{s}^{2}$ \\
\hline$\theta$ & dimensionless temperature \\
\hline$\eta$ & similarity parameter \\
\hline$e r f c$ & complementary error function \\
\hline
\end{tabular}

